\title{
Approximating Conductive Ellipsoid Inductive Responses Using Static Quadrupole Moments
}

\author{
Torquil Smith \\ Lawrence Berkeley National Laboratory \\ Berkeley, California 94720
}

This work was supported by the U.S. Department of Energy Of.ce of Management, Budget, and Evaluation under Contract Number DE-AC02-05CH11231 and the U.S. Department of Defense under the Strategic Environmental Research and Development Program Project 1225. 


\section{DISCLAIMER}

This document was prepared as an account of work sponsored by the United States Government. While this document is believed to contain correct information, neither the United States Government nor any agency thereof, nor The Regents of the University of California, nor any of their employees, makes any warranty, express or implied, or assumes any legal responsibility for the accuracy, completeness, or usefulness of any information, apparatus, product, or process disclosed, or represents that its use would not infringe privately owned rights. Reference herein to any specific commercial product, process, or service by its trade name, trademark, manufacturer, or otherwise, does not necessarily constitute or imply its endorsement, recommendation, or favoring by the United States Government or any agency thereof, or The Regents of the University of California. The views and opinions of authors expressed herein do not necessarily state or reflect those of the United States Government or any agency thereof or The Regents of the University of California. 


\section{Introduction}

Smith and Morrison (2006) developed an approximation for the inductive response of conducting magnetic (permeable) spheroids (e.g., steel spheroids) based on the inductive response of conducting magnetic spheres of related dimensions. Spheroids are axially symmetric objects with elliptical cross-sections along the axis of symmetry and circular cross sections perpendicular to the axis of symmetry. Spheroids are useful as an approximation to the shapes of unexploded ordnance (UXO) for approximating their responses. Ellipsoids are more general objects with three orthogonal principal axes, with elliptical cross sections along planes normal to the axes. Ellipsoids reduce to spheroids in the limiting case of ellipsoids with cross-sections that are in fact circles along planes normal to one axis. Parametrizing the inductive response of unknown objects in terms of the response of an ellipsoid is useful as it allows fitting responses of objects with no axis of symmetry, in addition to fitting the responses of axially symmetric objects. It is thus more appropriate for fitting the responses of metal scrap to be distinguished electromagnetically from unexploded ordnance. Here the method of Smith and Morrison (2006) is generalized to the case of conductive magnetic ellipsoids, and a simplified form used to parametrize the inductive response of isolated objects. The simplified form is developed

for the case of non-uniform source fields, for the first eight terms in an ellipsoidal harmonic decomposition of the source fields, allowing limited corrections for source field geometry beyond the common assumption of uniform source fields. 


\section{Ellipsoidal Coordinates}

The equation for an ellipsoid with semi-axes a', b', and c' in the $\hat{\mathbf{x}}, \hat{\mathbf{y}}$ and $\hat{\mathbf{z}}$ directions is

$$
\frac{x^{2}}{a^{\prime 2}}+\frac{y^{2}}{b^{\prime 2}}+\frac{z^{2}}{c^{\prime 2}}=1
$$

The equation for ellipsoidal coordinates $\xi_{1}, \xi_{2}$, and $\xi_{3}$ is

$$
\frac{x^{2}}{\xi^{2}-a^{2}}+\frac{y^{2}}{\xi^{2}-b^{2}}+\frac{z^{2}}{\xi^{2}}=1
$$

with $b<a$ by convention (Morse and Feshbach, 1957, or Hobson, 1931, with roles of $x$ and $z$ interchanged). Equation (2) is a cubic equation in $\xi^{2}$ when $x, y$, and $z$ are held fixed, so has three solutions $\xi^{2}$. The three coordinates $\xi_{1}, \xi_{2}$, and $\xi_{3}$ are such that $-b \leq \xi_{3} \leq b \leq\left|\xi_{2}\right| \leq a \leq\left|\xi_{1}\right|$. Solutions in $x, y$, and $z$ of Equation (2), for $\xi>a$ held fixed, define surfaces of constant $\xi_{1}$ which are confocal ellipsoids with short axis in the $\hat{\mathbf{x}}$ direction and long axis in the $\hat{\mathbf{z}}$ direction. That is, they give solutions of Equation (1) with $c^{\prime}=\xi_{1}, a^{\prime}=\sqrt{c^{\prime 2}-a^{2}}, b^{\prime}=\sqrt{c^{\prime 2}-b^{2}}$, where $a^{\prime} \leq b^{\prime} \leq c^{\prime}$. For computations involving a conducting ellipsoid with semi-axes $a^{\prime}, b^{\prime}$, and $c^{\prime}$, the ellipsoidal coordinate $\xi_{1}=c^{\prime}$ delimits the conducting ellipsoid, with $\xi_{1}$ values $a \leq\left|\xi_{1}\right|<c^{\prime}$ defining interior ellipsoidal surfaces and $\xi_{1}$ values $c^{\prime}<\left|\xi_{1}\right|$ defining exterior ellipsoidal surfaces. The limiting case ellipsoid with $\xi_{1}= \pm a$ corresponds to a flattened oval in the $y-z$ plane with zero thickness in the $\hat{\mathbf{x}}$ direction. Holding one of the other two ellipsoidal coordinates $\xi_{2}$ or $\xi_{3}$ constant defines (hyperboloid) surfaces which are orthogonal to each other and to the ellipsoids of constant $\xi_{1}$. Coordinate $\xi_{2}$ has two branches joined at $\xi_{2}=a$, and $\xi_{3}$ two joined at $\xi_{3}=b$ (Hobson, 1931). An ellipsoidal coordinate system is determined by the choice of principal ellipsoid axes $\hat{\mathbf{x}}, \hat{\mathbf{y}}$, and $\hat{\mathbf{z}}$, and parameters $a$ and $b$. Coordinates $x, y$, and $z$ are given by

$$
x=\frac{s_{\mu}\left(\xi_{1}^{2}-a^{2}\right)^{1 / 2}\left(a^{2}-\xi_{2}^{2}\right)^{1 / 2}\left(a^{2}-\xi_{3}^{2}\right)^{1 / 2}}{a\left(a^{2}-b^{2}\right)^{1 / 2}},
$$




$$
\begin{gathered}
y=\frac{s_{v}\left(\xi_{1}^{2}-b^{2}\right)^{1 / 2}\left(\xi_{2}^{2}-b^{2}\right)^{1 / 2}\left(b^{2}-\xi_{3}^{2}\right)^{1 / 2}}{b\left(a^{2}-b^{2}\right)^{1 / 2}} . \\
z=\frac{\xi_{1} \xi_{2} \xi_{3}}{a b},
\end{gathered}
$$

where $s_{\mu}= \pm 1=\operatorname{sign}(x)$ according to the branch of $\xi_{2}, s_{v}= \pm 1=\operatorname{sign}(y)$ according to the branch of $\xi_{3}$, and $\operatorname{sign}\left(\xi_{1}\right)=\operatorname{sign}(z)$.

Ellipsoidal coordinates are useful in electromagnetic induction problems in ellipsoidal objects at frequencies (or times) at which currents in the medium containing the ellipsoid object can be neglected. At these frequencies (times) the magnetic field outside the object is curl free (as well as divergence free) so can be parametrized as the gradient of a potential satisfying Laplace's equation. In ellipsoidal coordinates, solutions of Laplace's equation are separable; they can be written in terms of products of functions $E_{m}^{p}(\xi)$ and $F_{m}^{p}(\xi)$ of the three ellipsoidal coordinates: $E_{m}^{p}\left(\xi_{1}\right) E_{m}^{p}\left(\xi_{2}\right) E_{m}^{p}\left(\xi_{3}\right)$, or $F_{m}^{p}\left(\xi_{1}\right) E_{m}^{p}\left(\xi_{2}\right) E_{m}^{p}\left(\xi_{3}\right)$, where $E_{m}^{p}(\xi)$ and $F_{m}^{p}(\xi)$ are Lamè functions of the first and second kind, and $m$ and $p$ are indices which distinguish the different Lamè functions (e.g., Morse and Feshbach, 1953). Lamè functions of the first kind $E_{m}^{p}(\xi)$ are smooth and bounded for $\xi \rightarrow a$ (e.g., at the origin of $x, y$ and $z$ ) and, except $E_{0}^{0}(\xi)$ which is constant, increase without bound for $\xi \rightarrow \infty$, so they correspond to external sources, and Lamè functions of the second kind $F_{m}^{p}(\xi)$ increase without bound for $\xi \rightarrow a$, and vanish as $\xi \rightarrow \infty$, so those correspond to responses of the ellipsoids.

\section{Sphere Ellipsoid Response Approximation}

For axially symmetric objects (e.g., a spheroids), axial and transverse (equivalent dipole) polarizability responses $m_{a x}(t)$ and $m_{t}(t)$ give the strength, as a function of time $(t)$, of magnetic dipole moments in axial and transverse directions, induced by uniform (primary) magnetic fields of unit nominal amplitude in those directions, with a specified time function of primary magnetic field variation (e.g., Smith and Morrison, 2004). 
Strictly speaking, polarizabilities $m_{a x}(t)$ and $m_{t}(t)$ should have dimensions of Amp- $\mathrm{m}^{2} /$ $(A m p / m)=m^{3}$. However, since induction coils are sensitive to the rate of change of magnetic field, we let $m_{a x}(t)$ and $m_{t}(t)$ represent the time rate of change of polarizabilities, which have dimensions $\mathrm{m}^{3} / \mathrm{s}$, but will refer to them as 'polarizabilities'. Smith and Morrison (2006) approximate the axial and transverse equivalent dipole polarizability responses of conductive magnetic (permeable) spheroids using the polarizability responses of spheres of related sizes;

$$
\begin{gathered}
m_{a x}(t)=v_{a x} m_{\operatorname{sphere}\left(c_{e f f}\right)}(t) \\
m_{t}(t)=v_{t} m_{\operatorname{sphere}\left(b_{e f f}\right)}(t)
\end{gathered}
$$

Letting $c^{\prime}$ be the axial semi-diameter of the spheroid, and $b^{\prime}$ be the transverse semi-diameter of the spheroid, the transverse polarizability response of the spheroid is approximated as proportional to the polarizability response $m_{\text {sphere }\left(b_{\text {eff }}\right)}(t)$ of a sphere of the transverse semi-diameter $\left(b_{e f f} \equiv b^{\prime}\right)$, and the axial response is approximated as proportional to the response $m_{\text {sphere }\left(c_{e f f}\right)}(t)$ of a sphere of radius $c_{e f f}$, where for prolate spheroids $\left(c^{\prime}>b^{\prime}\right)$, $c_{e f f}=c^{\prime}$ and for oblate spheroids $\left(c^{\prime}<b^{\prime}\right), c_{e f f}=\sqrt{\left(c^{\prime 2}+b^{\prime} c^{\prime}\right) / 2}$, with the latter formula found empirically. This approximation will be denoted a sphere-spheroid approximation. Here we have written the approximation in terms of semi-diameters (radii) instead of diameters to be consistent with semi-axes used to characterize ellipsoids and ellipsoidal coordinates. Proportionality constants $v_{a x}$ and $v_{t}$ are ratios of differences of polarizabilities at high and low frequency limits;

$$
\begin{gathered}
v_{a x}=\frac{\left.m_{a x}^{(\text {spheroid })}\right|_{\omega=\infty}-\left.m_{a x}^{(\text {spheroid })}\right|_{\omega=0}}{\left.m_{\text {sphere }\left(c_{\text {eff }}\right)}\right|_{\omega=\infty}-m_{\text {sphere }\left(c_{\text {eff }}\right)_{\omega=0}}}, \\
v_{t}=\frac{\left.m_{t}^{\text {(spheroid })}\right|_{\omega=\infty}-\left.m_{t}^{(\text {spheroid })}\right|_{\omega=0}}{\left.m_{\text {sphere }\left(b_{\text {eff }}\right)}\right|_{\omega=\infty}-\left.m_{\text {sphere }\left(b_{\text {eff }}\right)}\right|_{\omega=0}},
\end{gathered}
$$

where the high and low frequency limit polarizabilities are given by Smith and Morrison 
(2006) and are readily calculated. Generalization to ellipsoids is straight forward. Prolate spheroids are the limiting case of ellipsoids with minor semi-axis length approaching their intermediate semi-axis length $\left(a^{\prime} \rightarrow b^{\prime}\right)$ with $\hat{\mathbf{z}}$ as their axis of symmetry. Oblate spheroids are the limiting case of ellipsoids with major semi-axis approaching their intermediate semi-axis length $\left(c^{\prime} \rightarrow b^{\prime}\right)$ with $\hat{\mathbf{x}}$ as their axis of symmetry. We approximate the polarizability response of conducting magnetic ellipsoid to uniform magnetic source fields (of a given time dependence) in the ellipsoid principal directions as

$$
\begin{aligned}
& m_{x}(t)=v_{x} m_{\text {sphere }\left(a_{e f f}\right)}(t), \\
& m_{y}(t)=v_{y} m_{\text {sphere }\left(b_{e f f}\right)}(t), \\
& m_{z}(t)=v_{z} m_{\text {sphere }\left(c_{e f f}\right)}(t),
\end{aligned}
$$

where

$$
v_{w}=\frac{\left.m_{w}^{(\text {ellipsoid })}\right|_{\omega=\infty}-\left.m_{w}^{(\text {ellipsoid })}\right|_{\omega=0}}{m_{\text {sphere }\left.\left(o_{\text {eff }}\right)\right|_{\omega=\infty}-\left.m_{\text {sphere }\left(o_{\text {eff }}\right)}\right|_{\omega=0}}},
$$

where $w$ stands for one of $x, y$, or $z$, and $o_{\text {eff }}$ stands for the corresponding choice among $a_{\text {eff }}, b_{\text {eff }}$, and $c_{\text {eff }}$. For ellipsoids, the appropriate effective sphere dimensions are given by

$$
a_{\text {eff }}=\left(\frac{a^{\prime 2}+a^{\prime} b^{\prime}}{2}\right)^{1 / 2}, \quad b_{e f f}=b^{\prime}, \quad c_{e f f}=c^{\prime},
$$

where, as before, $a^{\prime}, b^{\prime}$, and $c^{\prime}$ are the semi-axes of the ellipsoid. Effective sphere semidiameters (8) have been chosen to be consistent with those used for prolate and oblate spheroids, after allowing for the difference in symmetry axis between prolate and oblate spheroid treated as limiting cases of ellipsoids with $a^{\prime} \leq b^{\prime} \leq c^{\prime}$. Formula (7) for $v_{x}, v_{y}$ and $v_{z}$ requires the zero frequency and high frequency limit uniform source field 
polarizabilities for the ellipsoid in source fields oriented in the $\hat{\mathbf{x}}, \hat{\mathbf{y}}$, and $\hat{\mathbf{z}}$ directions respectively. These are derived from solutions of Laplace's equation in ellipsoidal coordinates for an external field that is the gradient of a potential varying in proportion to $x$, $y$, or $z$.

Ellipsoidal Harmonics and the Solution of Laplace's Equation in Ellipsoidal Coordinates

As noted above, solutions of Laplace's equation in ellipsoidal coordinates can be expressed in terms of products of Lamè functions of ellipsoidal coordinates,

$$
E_{m}^{p}\left(\xi_{1}\right) E_{m}^{p}\left(\xi_{2}\right) E_{m}^{p}\left(\xi_{3}\right)
$$

for external sources, and

$$
F_{m}^{p}\left(\xi_{1}\right) E_{m}^{p}\left(\xi_{2}\right) E_{m}^{p}\left(\xi_{3}\right)
$$

for internal sources. Forms (9) and (10) are known as external and internal ellipsoidal harmonics. The zero frequency limit induction problem is solved using a term of each form outside the ellipsoid, and a single term of form (9) inside the ellipsoid, that is,

$$
\begin{array}{ll}
\phi=\left[\alpha_{0} E_{m}^{p}\left(\xi_{1}\right)+\beta_{0} F_{m}^{p}\left(\xi_{1}\right)\right] E_{m}^{p}\left(\xi_{2}\right) E_{m}^{p}\left(\xi_{3}\right), & \text { outside, } \xi_{1} \geq \xi_{0}, \\
\phi=\alpha_{1} E_{m}^{p}\left(\xi_{1}\right) E_{m}^{p}\left(\xi_{2}\right) E_{m}^{p}\left(\xi_{3}\right), & \text { inside, } \xi_{1} \leq \xi_{0} .
\end{array}
$$

Letting $\xi_{1}=\xi_{0}$ be the $\xi_{1}$ coordinate of the ellipsoid/exterior interface, the zero frequency limit problem is to match $\mathbf{H} \times \hat{\mathbf{n}}$ and $\mathbf{B} \cdot \hat{\mathbf{n}}$ at $\xi_{1}=\xi_{0}$, where $\hat{\mathbf{n}}$ is the surface normal vector (in the $\xi_{1}$ direction). Of course $\mathbf{B}=\mu \mathbf{H}=\mu \nabla \phi$. Letting magnetic permeabilities be $\mu=\mu_{0}$ outside and $\mu=\mu_{1}$ inside the ellipsoid, this yields

$$
\left.\beta_{0}\right|_{\omega=0}=\left.\alpha_{0}\right|_{\omega=0}\left(\mu_{r}-1\right)\left(\frac{F^{\prime}\left(\xi_{0}\right)}{E^{\prime}\left(\xi_{0}\right)}-\mu_{r} \frac{F\left(\xi_{0}\right)}{E\left(\xi_{0}\right)}\right)^{-1}
$$

where ' denotes differentiation, $\mu_{r} \equiv \mu_{1} / \mu_{0}$, and indices $p$ and $m$ have been elided. 
The high frequency limit induction problem is the same as the low frequency limit problem with $\mathbf{B} \cdot \hat{\mathbf{n}}=0$ set at $\xi_{1}=\xi_{0}$. Solution of this case results in $\left.\alpha_{0}\right|_{\omega=\infty}$ and $\left.\beta_{0}\right|_{\omega=\infty}$ obeying Equation (12) in the limit of $\mu_{r} \rightarrow 0$, that is,

$$
\left.\beta_{0}\right|_{\omega=\infty}=-\left.\alpha_{0}\right|_{\omega=\infty} \frac{E^{\prime}\left(\xi_{0}\right)}{F^{\prime}\left(\xi_{0}\right)}
$$

Equations (12) and (13) solve low frequency and high frequency limit problems for external sources arising from a single external ellipsoidal harmonic term.

Lamè functions $E_{m}^{p}(\xi)$ and $F_{m}^{p}(\xi)$ depend on coordinate system parameters $a$ and $b$, which are constant for a given ellipsoidal coordinate system, so are omitted as arguments of $E_{m}^{p}$ and $F_{m}^{p}$. Following Morse and Feshbach (1957), the first few $E_{m}^{p}(\xi)$ are

$$
\begin{gathered}
E_{0}^{0}(\xi) \equiv 1, \\
E_{1}^{0}(\xi) \equiv \xi, \quad E_{1}^{1}(\xi) \equiv\left(\xi^{2}-a^{2}\right)^{1 / 2}, \quad E_{1}^{2}(\xi) \equiv\left(\xi^{2}-b^{2}\right)^{1 / 2} \\
E_{2}^{2}(\xi) \equiv \xi\left(\xi^{2}-a^{2}\right)^{1 / 2}, \quad E_{2}^{3}(\xi) \equiv \xi\left(\xi^{2}-b^{2}\right)^{1 / 2}, \quad E_{2}^{4}(\xi) \equiv\left(\xi^{2}-a^{2}\right)^{1 / 2}\left(\xi^{2}-b^{2}\right)^{1 / 2}, \\
E_{0}^{2}(\xi) \equiv \xi^{2}-\frac{1}{3}\left(a^{2}+b^{2}+d^{2}\right), \text { and } \quad E_{2}^{1}(\xi) \equiv \xi^{2}-\frac{1}{3}\left(a^{2}+b^{2}-d^{2}\right)
\end{gathered}
$$

where

$$
d^{2} \equiv\left[\left(a^{2}-b^{2}\right)^{2}+a^{2} b^{2}\right]^{1 / 2}
$$

Products of these form the first few external ellipsoidal harmonics, which can often be scaled to give simple multiples of coordinates $x, y$ and $z$;

$$
x=\frac{-\operatorname{sign}\left(\xi_{2}\right)}{a \sqrt{a^{2}-b^{2}}} E_{1}^{1}\left(\xi_{1}\right) E_{1}^{1}\left(\xi_{2}\right) E_{1}^{1}\left(\xi_{3}\right),
$$




$$
\begin{gathered}
y=\frac{i \operatorname{sign}\left(\xi_{1}\right)}{b \sqrt{a^{2}-b^{2}}} E_{1}^{2}\left(\xi_{1}\right) E_{1}^{2}\left(\xi_{2}\right) E_{1}^{2}\left(\xi_{3}\right), \\
z=\frac{\operatorname{sign}\left(\xi_{1}\right) \operatorname{sign}\left(\xi_{2}\right)}{a b} E_{1}^{0}\left(\xi_{1}\right) E_{1}^{0}\left(\xi_{2}\right) E_{1}^{0}\left(\xi_{3}\right), \\
y z=\frac{i \operatorname{sign}\left(\xi_{2}\right)}{a b^{2} \sqrt{a^{2}-b^{2}}} E_{2}^{3}\left(\xi_{1}\right) E_{2}^{3}\left(\xi_{2}\right) E_{2}^{3}\left(\xi_{3}\right), \\
x z=\frac{-\operatorname{sign}\left(\xi_{1}\right)}{a^{2} \sqrt{a^{2}-b^{2}}} E_{2}^{2}\left(\xi_{1}\right) E_{2}^{2}\left(\xi_{2}\right) E_{2}^{2}\left(\xi_{3}\right), \\
x y=\frac{-i \operatorname{sign}\left(\xi_{1}\right) \operatorname{sign}\left(\xi_{2}\right)}{a b \sqrt{a^{2}-b^{2}}} E_{2}^{4}\left(\xi_{1}\right) E_{2}^{4}\left(\xi_{2}\right) E_{2}^{4}\left(\xi_{3}\right) .
\end{gathered}
$$

However, external harmonics based on $E_{0}^{2}$ and $E_{2}^{1}$ are weighted sums of $x^{2}, y^{2}$, and $z^{2}$;

$$
E_{0}^{2}\left(\xi_{1}\right) E_{0}^{2}\left(\xi_{2}\right) E_{0}^{2}\left(\xi_{3}\right)=\alpha_{x} x^{2}+\alpha_{y} y^{2}+\alpha_{z} z^{2}+\alpha_{c}
$$

where

$$
\begin{gathered}
\alpha_{x}=\left(a^{2}+b^{2}+d^{2}\right)\left(a^{2}-2 b^{2}+d^{2}\right) / 9 \\
\alpha_{y}=\left(a^{2}+b^{2}+d^{2}\right)\left(b^{2}-2 a^{2}+d^{2}\right) / 9 \\
\alpha_{z}=\left(a^{2}+b^{2}+d^{2}\right)\left(a^{2}+b^{2}-2 d^{2}\right) / 9 \\
\alpha_{c}=\left(a^{2}+b^{2}+d^{2}\right)\left[\left(a^{2}-b^{2}\right)^{2}-2 a^{2} b^{2}+\left(a^{2}+b^{2}\right) d^{2}\right] / 27,
\end{gathered}
$$

and

$$
E_{2}^{1}\left(\xi_{1}\right) E_{2}^{1}\left(\xi_{2}\right) E_{2}^{1}\left(\xi_{3}\right)=\alpha_{x}^{\prime} x^{2}+\alpha_{y}^{\prime} y^{2}+\alpha^{\prime} z^{2}+\alpha_{c}^{\prime}
$$

where $\alpha^{\prime}{ }_{x}, \alpha^{\prime}{ }_{y}, \alpha^{\prime}{ }_{z}$, and $\alpha^{\prime}{ }_{c}$, are given by formulas (17) with $\alpha^{\prime}$ substituted for $\alpha$ and $-d^{2}$ 
substituted for $d^{2}$. Lamè functions of the second kind are more complicated;

$$
\begin{aligned}
F_{m}^{p}(\xi) & =(2 m+1) E_{m}^{p}(\xi) \int_{\xi}^{\infty} \frac{d z}{\left(z^{2}-a^{2}\right)^{1 / 2}\left(z^{2}-b^{2}\right)^{1 / 2}\left[E_{m}^{p}(z)\right]^{2}} \\
& =(2 m+1) E_{m}^{p}(\xi) \int_{0}^{1 / \xi} \frac{d x}{\left(1-a^{2} x^{2}\right)^{1 / 2}\left(1-b^{2} x^{2}\right)^{1 / 2}\left[E_{m}^{p}(1 / x)\right]^{2}}
\end{aligned}
$$

The individual $F_{m}^{p}(\xi)$ can, in principal, be expressed in terms of elementary elliptic integrals. Some are given in appendix.

For an external source potential of a single ellipsoidal harmonic $E_{m}^{p}\left(\xi_{1}\right) E_{m}^{p}\left(\xi_{2}\right) E_{m}^{p}\left(\xi_{3}\right)$, solution of both limiting case boundary problems results in potentials within the ellipsoid of the same functional form [Equations (11)], For uniform source magnetic fields aligned with one of the ellipsoid axes the limiting case boundary problem solution has the internal magnetic field aligned with the same axis. For the low frequency limit boundary problem, the 'anomalous' fields outside the ellipsoid due to the presence of the ellipsoid (the $\beta_{0} F_{m}^{p}$ term in Equations (11)), are simply the magnetic fields of the induced static magnetization inside the ellipsoid $\mathbf{M}_{1}=\left(\mu_{1}-\mu_{0}\right) \nabla \phi$. Given Equations (12) and (13), the anomalous fields for the high frequency limit boundary problem are simply $\left.\beta_{0}\right|_{\omega=\infty} /\left.\beta_{0}\right|_{\omega=0}$ times these.

\section{A Simplified Higher Order Sphere Ellipsoid Approximation}

For a single external ellipsoidal harmonic source field, the solution on the inside of an ellipsoid has the same functional form as the source field, for both low frequency and high frequency limit solutions, hence one is tempted to make a similar approximation to the sphere-spheroid approximation for higher order ellipsoidal harmonic sources. Because of symmetry, none of the higher order ellipsoidal harmonics induce any net dipole moment in an ellipsoid, so their effects first show up in the induced quadrupole moments of the ellipsoid. In the case of the three linearly polarized source fields $\left(H_{x}, H_{y}\right.$ 
and $H_{z}$ ), the limiting ellipsoidal boundary value problems result in uniform magnetic dipole distributions in the direction of the inducing field throughout the ellipsoid, with net magnetic dipole density rates of

$$
\rho_{x} \equiv m_{x}(t) / V \quad, \quad \rho_{y} \equiv m_{y}(t) / V \quad, \quad \rho_{z}=m_{z}(t) / V
$$

per unit nominal inducing field in the $\hat{\mathbf{x}}, \hat{\mathbf{y}}$, and $\hat{\mathbf{z}}$ directions respectively, and $V=4 \pi a^{\prime} b^{\prime} c^{\prime} / 3$ is the volume of the ellipsoid. The computationally simplest approximation for the response to higher order ellipsoidal harmonic source fields, is that the ellipsoid response is proportional to the local magnetic field with the same proportionality factors $\rho_{x}, \rho_{y}$, and $\rho_{z}$ as it is for the local magnetic fields in these directions for linearly polarized source fields. Under this assumption, because of symmetry, $E_{2}^{3}(\xi)(\phi=y z)$, $E_{2}^{2}(\xi)(\phi=x z), E_{2}^{4}(\xi)(\phi=x y), E_{0}^{2}(\xi)$, and $E_{2}^{1}(\xi)$ sources result in no net induced dipole moment, and $E_{1}^{1}(\xi)(\phi=x), E_{1}^{2}(\xi)(\phi=y)$, and $E_{1}^{0}(\xi)(\phi=z)$ sources result in no net induced quadrupole moments. The same assumption leads to induced quadrupole moment rates of

$$
\begin{gathered}
Q_{y z}(t)=H_{y z}\left(c_{e f f}^{2} \rho_{y}+b_{e f f}^{2} \rho_{z}\right) V / 5, \\
Q_{x z}(t)=H_{x z}\left(c_{e f f}^{2} \rho_{x}+a_{e f f}^{2} \rho_{z}\right) V / 5, \\
Q_{x y}(t)=H_{x y}\left(b_{e f f}^{2} \rho_{x}+a_{e f f}^{2} \rho_{y}\right) V / 5, \\
Q_{u u}(t)=2 H_{u u}\left(o_{e f f}^{2} \rho_{u}\right) V / 5,
\end{gathered}
$$

for source fields $\mathbf{H}=H_{u w}(u \hat{\mathbf{w}}+w \hat{\mathbf{u}})$, with $u$ and $w$ being any of $x, y$, and $z$, $\hat{\mathbf{u}}$ and $\hat{\mathbf{w}}$ being unit vectors in the corresponding directions, and, according to the choice of $u, o_{e f f}$ being the corresponding choice from $a_{\text {eff }}, b_{\text {eff }}$, and $c_{\text {eff }}$. Defining the $q_{u w}$ quadrupole polarizability as being the $Q_{u w}$ quadrupole moment per $H_{u w}$ for a $\mathbf{H}=H_{u w}(u \hat{\mathbf{w}}+w \hat{\mathbf{u}})$ source field, then $q_{u w}=Q_{u w} / H_{u w}$. So, under the assumption of pointwise consistency between the ratios of primary fields in the ellipsoid axis direction and induced dipole 
moment densities in those directions within the ellipsoid, one can write the ellipsoid quadrupole polarizability rates in terms of the ellipsoid dipole polarizability rates, and the effective sphere dimensions $a_{\text {eff }}, b_{\text {eff }}$, and $c_{\text {eff }}$ for the ellipsoid;

$$
\begin{gathered}
q_{y z}(t)=\left(c_{e f f}^{2} m_{y}(t)+b_{e f f}^{2} m_{z}(t)\right) / 5 \\
q_{x z}(t)=\left(c_{e f f}^{2} m_{x}(t)+a_{e f f}^{2} m_{z}(t)\right) / 5 \\
q_{x y}(t)=\left(b_{e f f}^{2} m_{x}(t)+a_{e f f}^{2} m_{y}(t)\right) / 5, \\
q_{u u}(t)=2 o_{e f f}^{2} m_{u}(t) / 5
\end{gathered}
$$

where $u, w$, and $o_{\text {eff }}$ are as before. Quadrupole polarizability (rates) $q$ have the dimensions of Amp- $\mathrm{m}^{3} /\left(\mathrm{s}-\mathrm{Amp} / \mathrm{m}^{2}\right)=\mathrm{m}^{5} / \mathrm{s}$. If the equivalent dipole polarizabilities of an ellipsoid are known by any means, quadrupole polarizabilities can be computed (for assumed or known ellipsoid dimensions $a^{\prime}, b^{\prime}$ and $c^{\prime}$ ) using Equations (22), and corrections can be made for the effects of quadrupole moments due to source field gradients.

Under the above assumption, that quadrupole responses within the ellipsoid are proportional to the local source magnetic field with the same proportionalities as the dipole polarizability responses, the resulting quadrupole moments are linear in the principal polarizabilities, and the fields of those moments are similarly linear in the principal polarizabilities. Letting $\mathbf{B}_{i j}^{(a)}$ be the anomalous field measured at the $i$ 'th sensor for the $j$ 'th source, $\mathbf{B}_{i k}^{(d i p)}$ be the fields at the $i$ 'th receiver of a $\hat{\mathbf{e}}_{k}$ dipole located at the object center, $\mathbf{B}_{\text {imn }}^{(\text {quad })}$ be the fields at the $i$ 'th receiver of a $\hat{\mathbf{e}}_{m} \hat{\mathbf{e}}_{n}$ quadrupole located at the object cen$\operatorname{ter}\left(\right.$ where $\left.\hat{\mathbf{e}}_{1}=\hat{\mathbf{x}}, \hat{\mathbf{e}}_{2}=\hat{\mathbf{y}}, \hat{\mathbf{e}}_{3}=\hat{\mathbf{z}}\right), \mathbf{B}_{j}^{(s r c)}$ be the fields of the $j$ 'th source at signal maximum, at the object center, $\nabla \mathbf{B}_{j}^{(s r o t)}$ be the gradient in object principal coordinates of the fields of the $j$ 'th source at the object center, with components $\nabla B_{k l j}^{(s r o t)}$, and $\mathbf{O}$ be the rotation matrix from object principal coordinates to field coordinates, then the anomalous fields at the receiver due to the object can be written as 


$$
\mathbf{B}_{i j}^{(a)}=\sum_{k, l, n=1,3} \mathbf{B}_{i k}^{(d i p)} O_{k n} p_{n} O_{l n} B_{l j}^{(s r c)}+\sum_{k, l, m, n=1,3} \mathbf{B}_{i m n}^{(q u a d)} O_{m k} O_{n l} \nabla B_{k l j}^{(s r o t)} p_{k} r_{l}^{2} / 5,
$$

where $p_{1}=m_{x}(t), p_{2}=m_{y}(t), p_{3}=m_{z}(t), r_{1}^{2}=a_{e f f}^{2}, r_{2}^{2}=b_{e f f}^{2}$, and $r_{3}^{3}=c_{e f f}^{2}$. Expression (23) has been simplified recognizing that $\hat{\mathbf{e}}_{m} \hat{\mathbf{e}}_{n}$ quadrupoles and $\hat{\mathbf{e}}_{n} \hat{\mathbf{e}}_{m}$ quadrupoles are identical and that $\nabla B_{k l j}^{(s r o t)}=\nabla B_{l k j}^{(s r o t)}$.

\section{Inversion for Ellipsoid Effective Semi-axes}

The anomalous magnetic fields given by Equation (23) are linear in principal polarizabilities, granted that object center location, orientation (e.g., Euler angles), and effective semi-axis lengths $a_{e f f}, b_{e f f}$, and $c_{e f f}$ are known. We invert for these parameters using a general non-linear optimization method (Smith, et al, 1994, Smith and Morrison, 2005), at each step fitting the principal polarizabilities using linear methods. We minimize a robust loss function of the data residuals weighted inversely by their estimated errors, minimizing squared weighted residuals for absolute weighted residuals less than 1 and absolute weighted residuals for absolute weighted residuals greater than one (Huber weights, e.g., Huber, 1981).

The algorithm was tested on data collected at the U.S. Army Yuma Proving Ground calibration grid, in Arizona. Data from vertical $\left( \pm 90^{\circ}\right)$ and dipping $\left( \pm 45^{\circ}\right) 81 \mathrm{~mm}(0.49 \mathrm{~m}$ long) and $155 \mathrm{~mm}(0.87 \mathrm{~m}$ long) UXO were inverted as these ordnance were sufficiently large and shallow that source gradient effects were expected. Results using ellipsoidal quadrupole polarizabilities are compared against results from inversion for object depth and polarizabilities without including ellipsoidal quadrupole polarizabilities in Tables I, II, and III. As azimuth is undefined for a vertical object, azimuth estimates for vertical UXO have been omitted. 


$\begin{array}{lcccc}\text { UXO } & \text { Dip (deg.) } & \text { Depth }(\mathrm{m}) & \begin{array}{c}\text { Est. Depth }(\mathrm{m}) \\ \text { w/o quadr. Polariz. }\end{array} & \begin{array}{c}\text { Est. Depth }(\mathrm{m}) \\ \text { w/ quadr. Polariz. }\end{array} \\ 81 \mathrm{~mm} & -45 & 0.87 & 0.80 & 0.96 \\ 81 \mathrm{~mm} & 45 & 0.87 & 0.83 & 0.83 \\ 81 \mathrm{~mm} & -90 & 0.95 & 0.74 & 0.80 \\ 81 \mathrm{~mm} & 90 & 0.95 & 0.83 & 0.92 \\ 155 \mathrm{~mm} & -45 & 1.26 & 1.13 & 1.14 \\ 155 \mathrm{~mm} & 45 & 1.26 & 1.09 & 1.15 \\ 155 \mathrm{~mm} & -90 & 1.39 & 1.22 & 1.28 \\ 155 \mathrm{~mm} & 90 & 1.39 & 1.16 & 1.30\end{array}$

Table I. Estimated depths below the Berkeley UXO Discriminator (BUD), for $81 \mathrm{~mm}$ and $155 \mathrm{~mm}$ UXO with and without ellipsoid quadrupole polarizabilities.

$\begin{array}{ccccc}\text { UXO } & \text { Depth (m) } & \text { Dip (deg.) } & \begin{array}{c}\text { Est. Dip (deg.) } \\ \text { w/o quadr. Polariz. }\end{array} & \begin{array}{c}\text { Est. Dip (deg.) } \\ \text { w/ quadr. Polariz. }\end{array} \\ 81 \mathrm{~mm} & 0.87 & -45 & -27.2 & -28.0 \\ 81 \mathrm{~mm} & 0.87 & 45 & 53.3 & 52.6 \\ 81 \mathrm{~mm} & 0.95 & -90 & -83.7 & -85.0 \\ 81 \mathrm{~mm} & 0.95 & 90 & 77.5 & 77.2 \\ 155 \mathrm{~mm} & 1.26 & -45 & -22.4 & -57.2 \\ 155 \mathrm{~mm} & 1.26 & 45 & 23.4 & 30.0 \\ 155 \mathrm{~mm} & 1.39 & -90 & -46.9 & -84.0 \\ 155 \mathrm{~mm} & 1.39 & 90 & 84.1 & 85.8\end{array}$

Table II. Estimated dips $\left( \pm 180^{\circ}\right)$ for $81 \mathrm{~mm}$ and $155 \mathrm{~mm}$ UXO with and without ellipsoid quadrupole polarizabilities. 


$\begin{array}{lccccc}\text { UXO } & \text { Depth (m) } & \text { Dip (deg.) } & \text { Azim. (deg). } & \begin{array}{c}\text { Est. Azim. (deg.) } \\ \text { w/o quadr. Polariz. }\end{array} & \begin{array}{c}\text { Est. Azim. (deg.) } \\ \text { w/ quadr. Polariz. }\end{array} \\ 81 \mathrm{~mm} & 0.87 & -45 & 0 & 4.3 & 6.8 \\ 81 \mathrm{~mm} & 0.87 & 45 & 0 & -9.3 & -12.9 \\ 155 \mathrm{~mm} & 1.26 & -45 & 0 & 36.8 & 17.8 \\ 155 \mathrm{~mm} & 1.26 & 45 & 0 & -6.1 & -5.7\end{array}$

Table III. Estimated azimuths $\left( \pm 180^{\circ}\right)$ for $81 \mathrm{~mm}$ and $155 \mathrm{~mm}$ UXO with and without ellipsoid quadrupole polarizabilities.

Depths estimated without ellipsoid quadrupole polarizabilities have a root mean squared (rms) error of $0.15 \mathrm{~m}$, those estimated with ellipsoid quadrupole polarizabilities have 0.10 $\mathrm{m}$ rms error. Dip estimates have a $20.8^{\circ}$ rms error without ellipsoid quadrupole polarizabilities, and a $11.0^{\circ}$ rms error with ellipsoid quadrupole polarizabilities. Azimuth estimates have $19.3^{\circ}$ and $11.9^{\circ}$ rms errors without and with ellipsoid quadrupole polarizabilities, respectively. In summary, using ellipsoid polarizabilities when estimating position, dip, azimuth, and polarizabilities, for objects where source gradients are significant along the length of the object improves the estimates of position, dip and azimuth.

Comparison of polarizability estimates is more difficult as true uniform field polarizabilities are not known a priori, but are estimated from measured data. Polarizability estimates made without ellipsoid quadrupole polarizabilities are shown in Figure 1, for the $81 \mathrm{~mm} \mathrm{UXO}$ in the four orientations of Tables I and II, while polarizability estimates made with ellipsoid quadrupole polarizabilities are shown in Figure 2. Similarly, polarizability estimates made without ellipsoid quadrupole for the $155 \mathrm{~mm}$ UXO in the same orientations are show in Figure 3, those made with ellipsoid quadrupole polarizabilities are shown in Figure 4. In Figures 1 and 2, and of Figures 3 and 4, the individual curves are more easily recognized as axially symmetric objects in the curves calculated without ellipsoid quadrupole polarizabilities (Figures 1 and 3), as there, the two minor 
polarizabilities match each other more closely. However, the polarizability estimates made with ellipsoid quadrupole polarizabilities match each other better between upward and downward orientations (negative and positive dips) for both the $81 \mathrm{~mm}$ UXO and the $155 \mathrm{~mm}$ UXO, than do the polarizability estimates made without ellipsoid quadrupole polarizabilities, suggesting that it may be easier to recognize the corrected curves as being due to the same UXO, so may be preferable for UXO model identification.

\section{Conclusion}

The quadrupole polarizabilities we fit here were based on assuming point wise consistency between the ratios of primary fields in the ellipsoid axis direction and induced dipole moment densities in those directions within the ellipsoid, between the cases of induction by uniform fields and induction by first order gradient fields. For UXO with significant source field gradients along their lengths, fitting ellipsoid quadrupole polarizabilities under that assumption simultaneous with standard dipole polarizabilities reduced the errors made in estimating object center position and object orientation, and resulted in polarizability curves that vary less with object orientation, at the expense of poorer agreement between a particular response's two minor (transverse) polarizabilities. A more elaborate ellipsoid sphere approximation for the higher order terms, scaling sphere responses to $y z, x z, x y$, and $x^{2}-z^{2}$ gradient fields, in a manner similar to Equations (6), to approximate ellipsoid responses to $y z, x z, x y, E_{0}^{2}(\xi)$, and $E_{2}^{1}(\xi)$ gradients, may be more accurate than the ellipsoid quadrupole polarizabilities estimated using the assumption of point wise consistency of induced dipole moment densities which were described here.

\section{Acknowledgement}

This work was supported by the U.S. Department of Energy Office of Management, Budget, and Evaluation under Contract Number DE-AC02-05CH11231 and the U.S. Department of Defense under the Strategic Environmental Research and Development Program Project 1225. 
Appendix: Lamè Functions of the Second Kind $F_{m}^{p}$ and Their Derivative

Lamè functions of the second kind involve elliptic integrals. Here pertinent $F_{m}^{p}$ are written in terms of elliptic integrals amenable to evaluation using published algorithms (e.g., Bulirsch, 1965a, 1965b, 1969).

$$
\begin{aligned}
& F_{1}^{0}(\xi)=3 \xi \int_{0}^{1 / \xi} \frac{x^{2} d x}{\left(1-a^{2} x^{2}\right)^{1 / 2}\left(1-b^{2} x^{2}\right)^{1 / 2}} \\
& F_{1}^{1}(\xi)=3\left(\xi^{2}-a^{2}\right)^{1 / 2} \int_{0}^{1 / \xi} \frac{x^{2} d x}{\left(1-a^{2} x^{2}\right)^{3 / 2}\left(1-b^{2} x^{2}\right)^{1 / 2}} \\
&=\frac{3\left(\xi^{2}-a^{2}\right)^{1 / 2}}{a^{2}}\left[\int_{0}^{1 / \xi} \frac{d x}{\left(1-a^{2} x^{2}\right)^{3 / 2}\left(1-b^{2} x^{2}\right)^{1 / 2}}-\int_{0}^{1 / \xi} \frac{x^{2}}{\left(1-a^{2} x^{2}\right)^{1 / 2}\left(1-b^{2} x^{2}\right)^{1 / 2}}\right] \\
& F_{1}^{2}(\xi)=3\left(\xi^{2}-b^{2}\right)^{1 / 2} \int_{0}^{1 / \xi} \frac{x^{2} d x}{\left(1-a^{2} x^{2}\right)^{1 / 2}\left(1-b^{2} x^{2}\right)^{3 / 2}} \\
& F_{2}^{2}(\xi)=5 \xi\left(\xi^{2}-a^{2}\right)^{1 / 2} \int_{0}^{1 / \xi} \frac{d x}{\left(1-a^{2} x^{2}\right)^{3 / 2}\left(1-b^{2} x^{2}\right)^{1 / 2}} \\
&=\frac{3\left(\xi^{2}-b^{2}\right)^{1 / 2}}{b^{2}}\left[\int_{0}^{1 / \xi} \frac{d(\mathrm{~A}-3)}{\left(1-a^{2} x^{2}\right)^{1 / 2}\left(1-b^{2} x^{2}\right)^{3 / 2}}-\int_{0}^{1 / \xi} \frac{d x}{\left(1-a^{2} x^{2}\right)^{1 / 2}\left(1-b^{2} x^{2}\right)^{1 / 2}}\right]
\end{aligned}
$$$$
=\frac{5 \xi\left(\xi^{2}-a^{2}\right)^{1 / 2}}{a^{4}}\left[\int_{0}^{1 / \xi} \frac{d x}{\left(1-a^{2} x^{2}\right)^{3 / 2}\left(1-b^{2} x^{2}\right)^{1 / 2}}+\int_{0}^{1 / \xi} \frac{\left(1-a^{2} x^{2}\right) d x}{\left(1-a^{2} x^{2}\right)^{1 / 2}\left(1-b^{2} x^{2}\right)^{1 / 2}}-2 \int_{0}^{1 / \xi} \frac{d x}{\left(1-a^{2} x^{2}\right)^{1 / 2}\left(1-b^{2} x^{2}\right)^{1 / 2}}\right]
$$ 


$$
F_{2}^{3}(\xi)=5 \xi\left(\xi^{2}-b^{2}\right)^{1 / 2} \int_{0}^{1 / \xi} \frac{x^{4} d x}{\left(1-a^{2} x^{2}\right)^{1 / 2}\left(1-b^{2} x^{2}\right)^{3 / 2}}
$$

$=\frac{5 \xi\left(\xi^{2}-b^{2}\right)^{1 / 2}}{b^{4}}\left[\int_{0}^{1 / \xi} \frac{d x}{\left(1-a^{2} x^{2}\right)^{1 / 2}\left(1-b^{2} x^{2}\right)^{3 / 2}}+\int_{0}^{1 / \xi} \frac{\left(1-b^{2} x^{2}\right) d x}{\left(1-a^{2} x^{2}\right)^{1 / 2}\left(1-b^{2} x^{2}\right)^{1 / 2}}-2 \int_{0}^{1 / \xi} \frac{d x}{\left(1-a^{2} x^{2}\right)^{1 / 2}\left(1-b^{2} x^{2}\right)^{1 / 2}}\right]$,

$$
F_{2}^{4}(\xi)=5\left(\xi^{2}-a^{2}\right)^{1 / 2}\left(\xi^{2}-b^{2}\right)^{1 / 2} \int_{0}^{1 / \xi} \frac{x^{4} d x}{\left(1-a^{2} x^{2}\right)^{3 / 2}\left(1-b^{2} x^{2}\right)^{3 / 2}}
$$

$=\frac{5\left(\xi^{2}-a^{2}\right)^{1 / 2}\left(\xi^{2}-b^{2}\right)^{1 / 2}}{a^{2} b^{2}}\left[\int_{0}^{1 / \xi} \frac{d x}{\left(1-a^{2} x^{2}\right)^{1 / 2}\left(1-b^{2} x^{2}\right)^{1 / 2}}+\frac{b^{2}}{a^{2}-b^{2}} \int_{0}^{1 / \xi} \frac{d x}{\left(1-a^{2} x^{2}\right)^{3 / 2}\left(1-b^{2} x^{2}\right)^{1 / 2}}\right.$

$$
\left.-\frac{a^{2}}{a^{2}-b^{2}} \int_{0}^{1 / \xi} \frac{d x}{\left(1-a^{2} x^{2}\right)^{1 / 2}\left(1-b^{2} x^{2}\right)^{3 / 2}}\right]
$$

$F_{0}^{2}(\xi)=\left[\xi^{2}-\left(a^{2}+b^{2}+d^{2}\right) / 3\right] \int_{0}^{1 / \xi} \frac{x^{4} d x}{\left(1-a^{2} x^{2}\right)^{1 / 2}\left(1-b^{2} x^{2}\right)^{1 / 2}\left[1-x^{2}\left(a^{2}+b^{2}+d^{2}\right) / 3\right]^{2}}(\mathrm{~A}-7)$

$$
=\frac{\xi^{2}-\alpha^{2}}{\alpha^{4}}\left[\left(1-\frac{1}{2 \alpha^{4} b_{0}}\right)_{0}^{1 / \xi} \frac{d x}{\left(1-a^{2} x^{2}\right)^{1 / 2}\left(1-b^{2} x^{2}\right)^{1 / 2}}+\frac{1}{2 \alpha^{4} b_{0}} \int_{0}^{1 / \xi} \frac{x^{2} d x}{\left(1-a^{2} x^{2}\right)^{1 / 2}\left(1-b^{2} x^{2}\right)^{1 / 2}}\right.
$$

$\left.-\frac{1}{2}\left(3+\frac{a^{2}}{\alpha^{2}-a^{2}}+\frac{b^{2}}{\alpha^{2}-b^{2}}\right)_{0}^{1 / \xi} \frac{d x}{\left(1-a^{2} x^{2}\right)^{1 / 2}\left(1-b^{2} x^{2}\right)^{1 / 2}\left(1-\alpha^{2} x^{2}\right)}+\frac{\left(\xi^{2}-a^{2}\right)^{1 / 2}\left(\xi^{2}-b^{2}\right)^{1 / 2}}{2 a^{2} b^{2} b_{0}\left(\xi^{3}-\alpha^{2} \xi\right)}\right]$,

where 


$$
\alpha^{2} \equiv\left(a^{2}+b^{2}+d^{2}\right) / 3 \quad, \quad b_{0} \equiv \frac{\left(\alpha^{2}-a^{2}\right)\left(\alpha^{2}-b^{2}\right)}{a^{2} b^{2} \alpha^{4}}
$$

$$
F_{2}^{1}(\xi)=5\left[\xi^{2}-\left(a^{2}+b^{2}-d^{2}\right) / 3\right] \int_{0}^{1 / \xi} \frac{x^{4} d x}{\left(1-a^{2} x^{2}\right)^{1 / 2}\left(1-b^{2} x^{2}\right)^{1 / 2}\left[1-x^{2}\left(a^{2}+b^{2}-d^{2}\right) / 3\right]^{2}}
$$

$$
=\frac{5\left(\xi^{2}-\beta^{2}\right)}{\beta^{4}}\left[\left(1-\frac{1}{2 \beta^{4} c_{0}}\right)^{1 / \xi} \int_{0}^{\left.1-a^{2} x^{2}\right)^{1 / 2}\left(1-b^{2} x^{2}\right)^{1 / 2}}+\frac{1}{2 \beta^{4} c_{0}} \int_{0}^{1 / \xi} \frac{x^{2} d x}{\left(1-a^{2} x^{2}\right)^{1 / 2}\left(1-b^{2} x^{2}\right)^{1 / 2}}\right.
$$

$$
\left.-\frac{1}{2}\left(3+\frac{a^{2}}{\beta^{2}-a^{2}}+\frac{b^{2}}{\beta^{2}-b^{2}}\right)_{0}^{1 / \xi} \frac{d x}{\left(1-a^{2} x^{2}\right)^{1 / 2}\left(1-b^{2} x^{2}\right)^{1 / 2}\left(1-\beta^{2} x^{2}\right)}+\frac{\left(\xi^{2}-a^{2}\right)^{1 / 2}\left(\xi^{2}-b^{2}\right)^{1 / 2}}{2 a^{2} b^{2} c_{0}\left(\xi^{3}-\beta^{2} \xi\right)}\right],
$$

where

$$
\beta^{2} \equiv\left(a^{2}+b^{2}-d^{2}\right) / 3, \quad c_{0} \equiv \frac{\left(\beta^{2}-a^{2}\right)\left(\beta^{2}-b^{2}\right)}{a^{2} b^{2} \beta^{4}} .
$$

Derivative $F_{m}^{\prime}{ }_{m}(\xi)$ can be expressed in terms of derivative ${E^{\prime}}_{m}^{p}(\xi)$ using Equation (19) and the fundamental theorem of calculus, yielding

$$
F_{m}^{\prime p}(\xi)=\frac{F_{m}^{p}(\xi)}{E_{m}^{p}(\xi)} E_{m}^{\prime p}(\xi)-\frac{(2 m+1)}{\left(\xi^{2}-a^{2}\right)^{1 / 2}\left(\xi^{2}-b^{2}\right)^{1 / 2} E_{m}^{p}(\xi)}
$$




\section{References}

Bulirsch, R., Numerical calculation of elliptic integrals and elliptic functions, Numer. Math., 7, 78-90, 1965a.

Bulirsch, R., Numerical calculation of elliptic integrals and elliptic functions, II, Numer. Math., 7, 353-354, 1965 b.

Bulirsch, R., Numerical calculation of elliptic integrals and elliptic functions, III, Numer. Math., 13, 305-315 1969.

Hobson, E.W., The Theory of Spherical and Ellipsoidal Harmonics, Cambridge Univ. Press, Cambridge, 1931. Reprinted by Chelsea Publishing Co., New York, 1955.

Huber, P.J., 1981, Robust Statistics, John Wiley and Sons, New York, 1979.

Morse, P.M., and Feshbach, H., Methods of Theoretical Physics, part II, McGraw-Hill, New York, 1953.

Smith, J.T., and Morrison, H.F., Estimating equivalent dipole polarizabilities for the inductive response of isolated conductive bodies. I.E.E.E. Trans. Geosci. Remote Sensing, 42, 1208-1214, 2004.

Smith, J.T., and Morrison, H.F., Optimizing receiver configurations for resolution of equivalent dipole polarizabilities in situ. I.E.E.E. Trans. Geosci. Remote Sensing, 43, 1490-1498, 2005.

Smith, J.T., and Morrison, H.F., Approximating spheroid inductive responses using spheres. Geophysics, 71, G21-G25, 2006.

T. Smith, W. Allan, and A. Schultz, Inversion of waveform data using an empirical distribution evolutionary algorithm, Eos, vol. 75, no. 44, p. 457, 1994. 

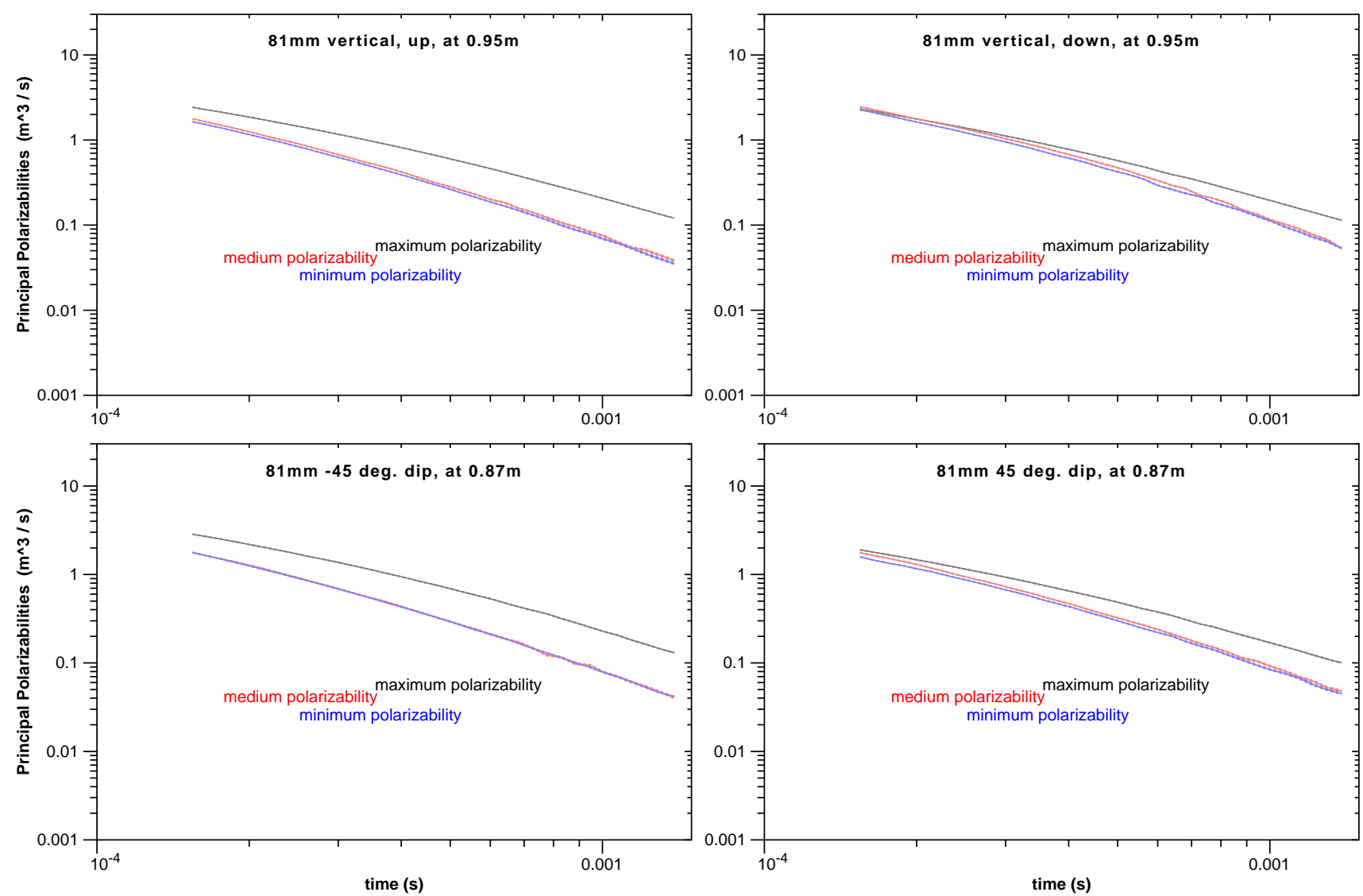

Figure (1). Polarizability estimates made without ellipsoid quadrupole polarizabilities for a $81 \mathrm{~mm}$ UXO (top left) oriented vertically, pointed up, centered 0.95 below the Berkeley UXO Discriminator (BUD), (top right) oriented vertically, pointed down, centered 0.95 below BUD, (bottom left) dipping $-45^{\mathrm{O}}$ centered 0.87 below BUD, (bottom right) dipping $45^{\circ}$, centered 0.87 below BUD. 

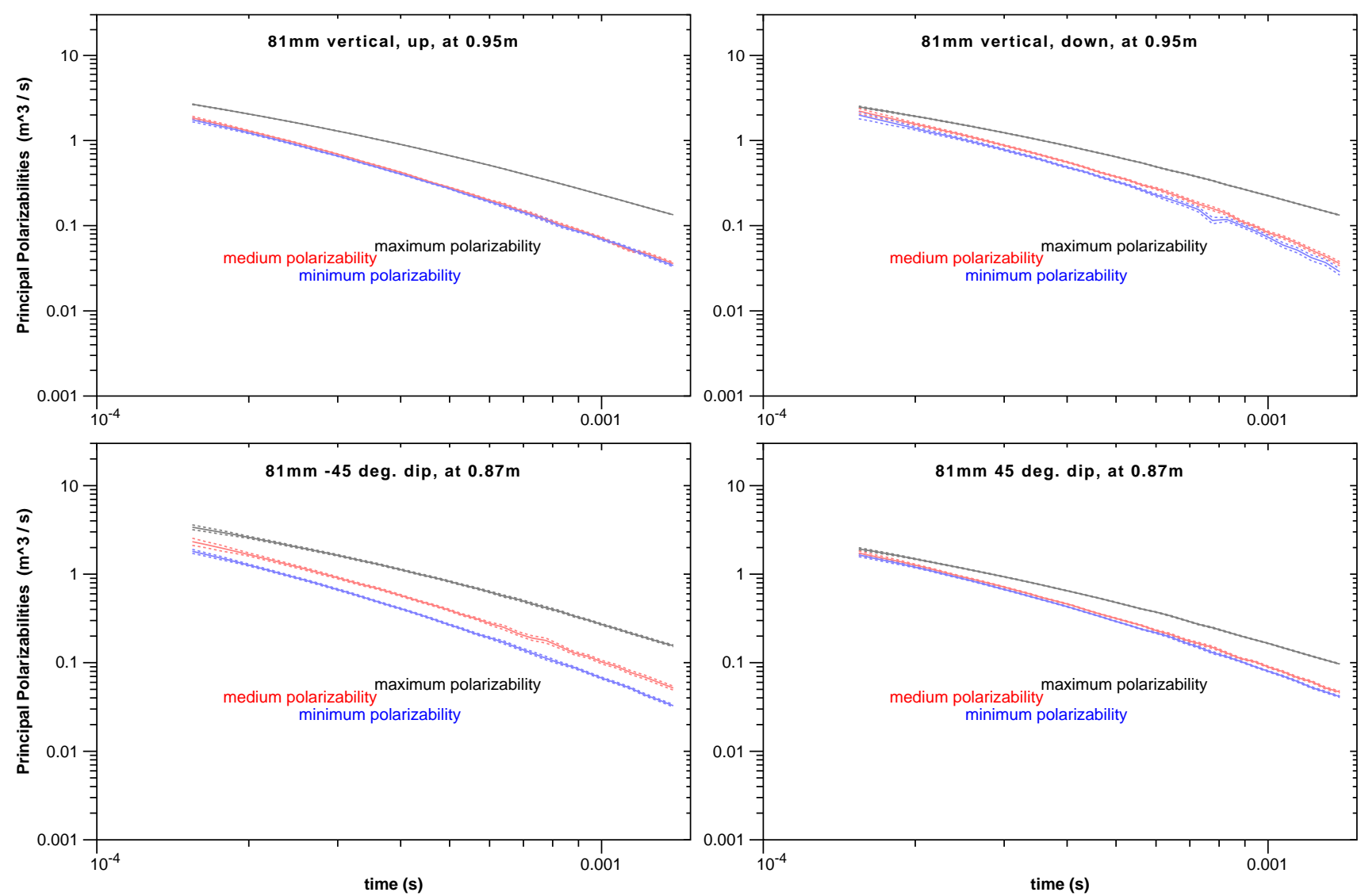

Figure (2). Polarizability estimates made with ellipsoid quadrupole polarizabilities for a 81 mm UXO (top left) oriented vertically, pointed up, centered $0.95 \mathrm{~m}$ below BUD, (top right) oriented vertically, pointed down, centered $0.95 \mathrm{~m}$ below BUD, (bottom left) dipping $-45^{\mathrm{O}}$ centered $0.87 \mathrm{~m}$ below BUD, (bottom right) dipping $45^{\circ}$, centered $0.87 \mathrm{~m}$ below BUD. 

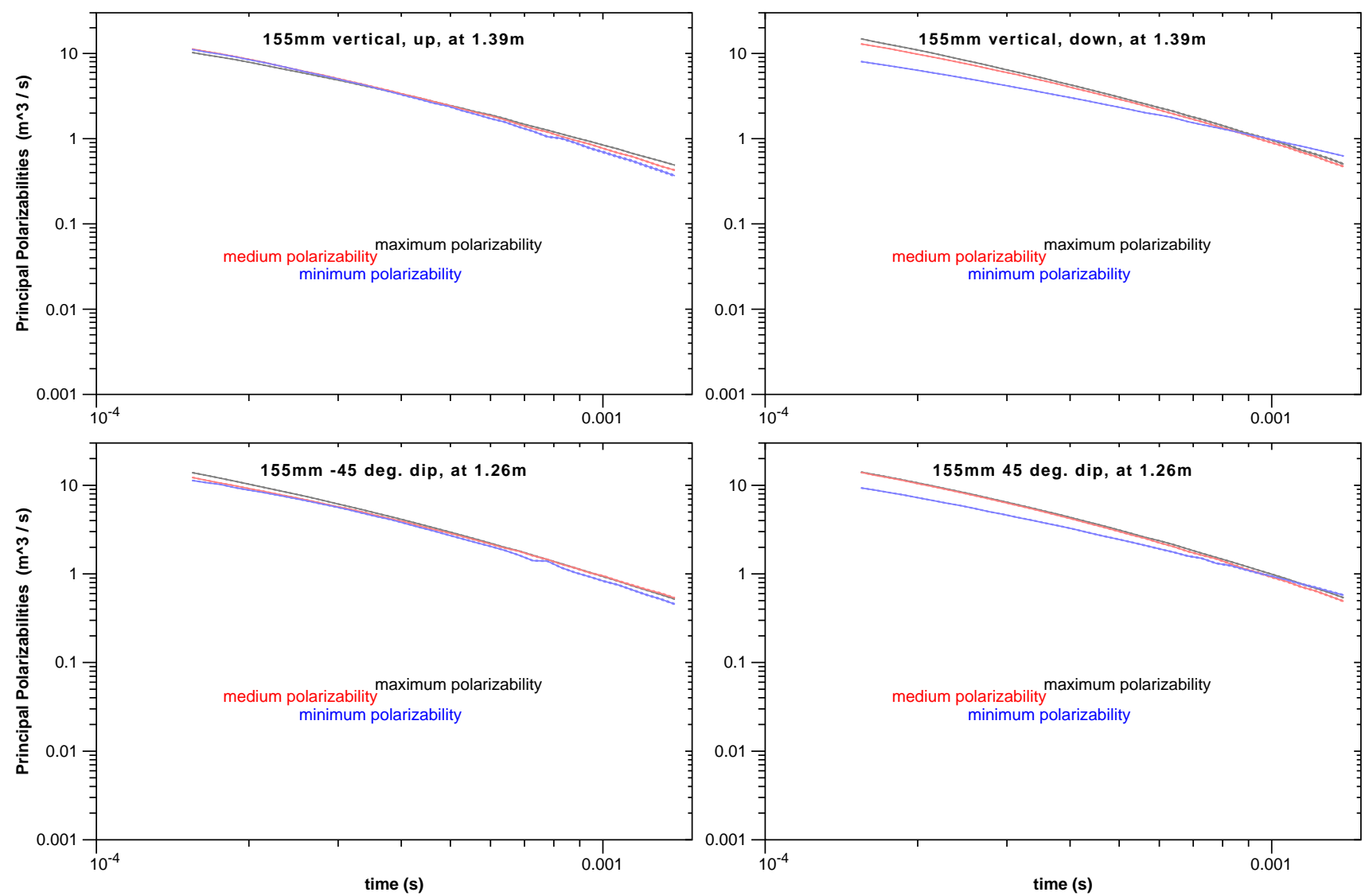

Figure (3). Polarizability estimates made without ellipsoid quadrupole polarizabilities for a $155 \mathrm{~mm}$ UXO (top left) oriented vertically, pointed up, centered $1.39 \mathrm{~m}$ below BUD, (top right) oriented vertically, pointed down, centered $1.39 \mathrm{~m}$ below bud, (bottom left) dipping $-45^{\circ}$, centered $1.26 \mathrm{~m}$ below BUD, (bottom right) dipping $45^{\circ}$, centered $1.26 \mathrm{~m}$ below BUD. 

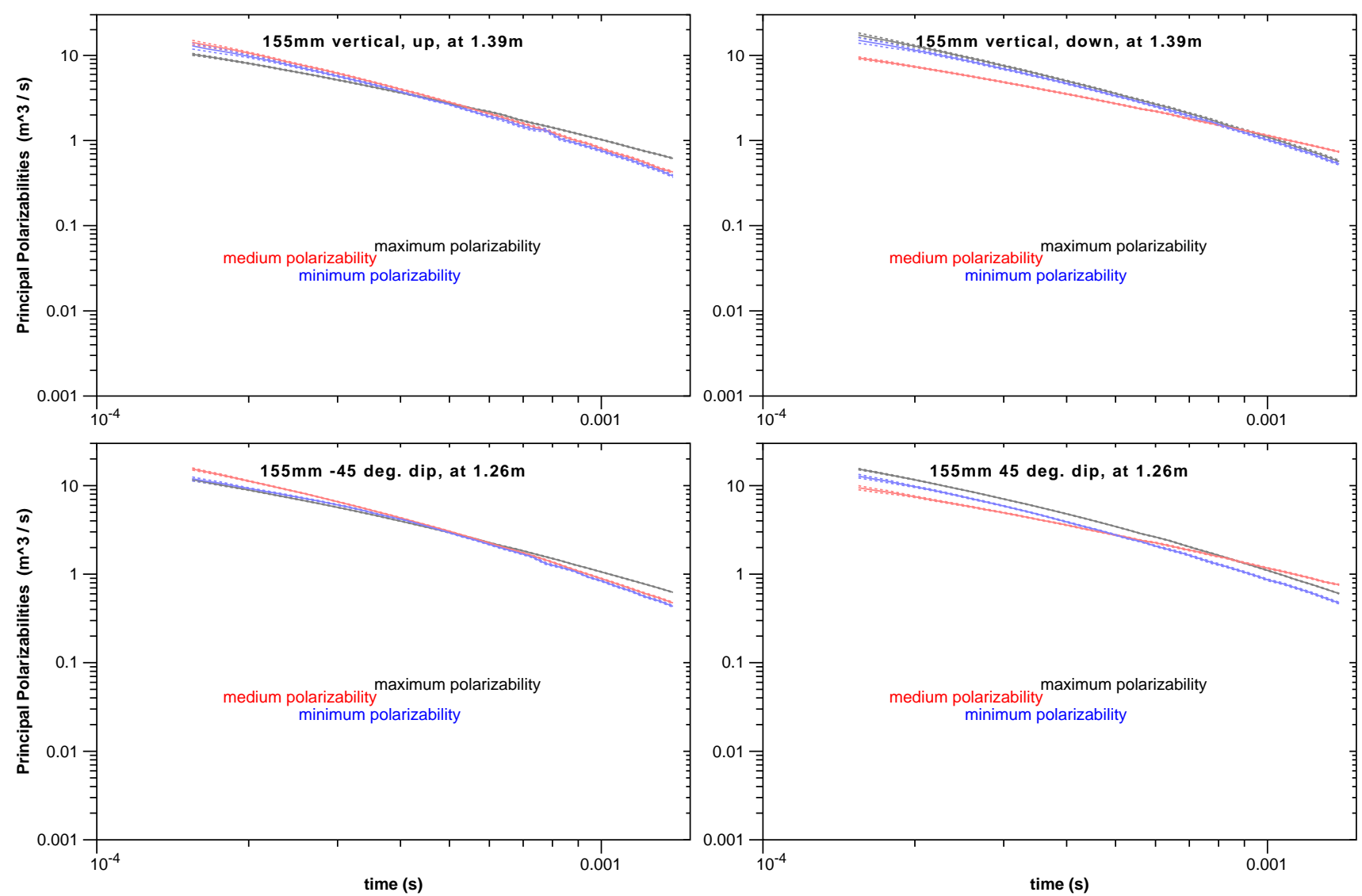

Figure (4). Polarizability estimates made with ellipsoid quadrupole polarizabilities for a 155 mm UXO (top left) oriented vertically, pointed up, centered $1.39 \mathrm{~m}$ below BUD, (top right) oriented vertically, pointed down, centered $1.39 \mathrm{~m}$ below bud, (bottom left) dipping $-45^{\circ}$, centered $1.26 \mathrm{~m}$ below BUD, (bottom right) dipping $45^{\circ}$, centered $1.26 \mathrm{~m}$ below BUD. 